\title{
Monetary Budget Constraint and Its Missing Problems
}

\author{
Shinji Miura \\ Independent, Gifu, Japan \\ Email: geppa gifu@yahoo.co.jp
}

Received 23 July 2015; accepted 8 September 2015; published 11 September 2015

Copyright (C) 2015 by author and Scientific Research Publishing Inc.

This work is licensed under the Creative Commons Attribution International License (CC BY). http://creativecommons.org/licenses/by/4.0/

\section{(c) () Open Access}

\begin{abstract}
This paper aims to inquire into an objectively authentic budget constraint in a monetary economy through showing two missing problems of the monetary budget constraint and their solutions. To start with, we show the first missing problem that money is "missing" if all agents expend their total budgets under the simple budget constraint. This problem shows that the simple budget constraint is inadequate as an objective monetary budget constraint. A deficiency of the simple budget constraint exists partly in that it does not reflect money circulation. To improve this deficiency, we incorporate the expenditure reflux formula into the simple constraint. The first missing problem is partially solved by the application of this reflux budget constraint, but another problem occurs. The new problem is that infinite expenditure is permitted under this constraint. This is the second missing problem. The second problem appears to be a variation of the solvability problem of the money circulation equation. Referring to the proof of the solvability, we incorporate a time irreversible disposal into the budget constraint. This irreversibility budget constraint brings us a provisional solution of the missing problems. However, it should not be called a perfect solution. We also examine the relationships between our research and two previous studies: the finance constraint and the cash-in-advance model.
\end{abstract}

\section{Keywords}

Budget Constraint, Money Circulation, Expenditure Reflux, Time Irrevesibility

\section{Introduction}

Much of contemporary economics is based on optimal decision making under the budget constraint. Since modern economies are monetary economies, a realistic budget constraint in a modern economy must be a monetary constraint. To consider the monetary budget constraint is the purpose of this paper. 
Some agents may recognize an objectively wrong constraint and make decisions based on this constraint. Therefore, when we think about a budget constraint, we need to distinguish between a constraint used by economic agents on the occasion of decision making subjectively and a constraint enforced on an agent objectively. The purpose of this paper is not to consider the former constraint but to consider the latter constraint in a monetary economy.

An objective constraint may not be used by agents, but it is worth being researched by the following two reasons. First, it is significant as normative research. If a budget constraint identified in this research is objectively valid, we may be able to provide material to improve decision making by agents. Moreover, it is also significant as positive research. An optimal solution derived by using an objectively wrong constraint may be unrealizable. We will show an example of this in the following section. In this case, a subjective decision which was once made must be modified. In order to judge whether the modification is avoidable or not, we should not ignore the objective constraint even when considering subjective decision making.

There are some notable previous studies in connection with the focus of this paper. First, we discuss two missing problems of the monetary budget constraint and show their solutions. After this, we will introduce and comment on the previous research efforts.

\section{Simple Budget Constraint and the First Missing Problem of the Monetary Budget Constraint}

To start with, we will clarify the basic framework of this paper. The framework is basically the same as that used by Miura [1] and Miura [2].

We decide that the target group of economic agents for description is called the relevant society ${ }^{1}$. There are various kinds of agents, such as individuals, families, corporations, governments, and so on. In economics, the budget constraint of consumers is discussed frequently, and that of governments is discussed occasionally ${ }^{2}$, but there is scant reference to the budget constraint of corporations. However, a corporation must expend money, thus its budget constraint also exists ${ }^{3}$. This paper discusses all the budget constraints of these agents, but does not consider the differences between types of agents; we treat them merely as agents in the group. We denote the relevant society by $N=\{1,2, \cdots, n\}$.

We decide that the target term for description is called the relevant term. We assume that the relevant term is finite length. This assumption is rewritten so that the relevant term always has a beginning and an end.

The sphere which satisfies both the relevant society and the relevant term is called the relevant space-time.

Furthermore, we do not distinguish between various types of money transfer, including purchase of commodities, payment of wages, finance relations, donations, and so on. Also, there are various kinds of money such as the dollar, pound, euro, yen, and so on. However, this paper assumes that money in the relevant space-time is of only one kind.

We define expenditure as transferring money to the relevant space-time, and revenue as money being transferred from the relevant space-time. There is a possibility that money transfer occurs between the relevant society and its outside, but transferring money to the outside is not called expenditure and money being transferred from the outside is not called revenue in this paper.

Moreover, we decide that the sources excluding revenue, such as possessions at the beginning of the relevant term, production and funds transferred from outside the relevant society, are collectively called the beginning money. Also, we decide that the results excluding expenditure, such as possessions at the end of the relevant term, disappearance and funds transferred to outside the relevant society, are collectively called the end money.

Let $X_{k}$ be the expenditure quantity of Agent $k ; Y_{k}$ be the revenue quantity of Agent $k ; \Psi_{k}$ be the quantity of the beginning money of Agent $k$; and $\Omega_{k}$ be the quantity of the end money of Agent $k$. By their economic meaning, they are all non-negative. That is, $X_{k} \geq 0, Y_{k} \geq 0, \Psi_{k} \geq 0, \Omega_{k} \geq 0$ holds for $\forall k \in N$.

Miura [1] confirms that the sum of gross source and the sum of gross result are equal in any agent. This proposition is named the law of gross disposal ${ }^{4}$. Using symbols, the law is denoted as follows:

\footnotetext{
${ }^{1}$ It is permitted that one element is either one agent or one group of agents, but we call it merely an agent.

${ }^{2}$ The idea of the government budget constraint seems to occur in the 1950s. Cf. Christ [3] p. 53.

${ }^{3}$ If profit is defined only by money transfer, we may be able to interpret that the budget constraint of corporations is included in the definition equation of profit.

${ }^{4}$ Cf. Miura [1] p. 190.
} 


$$
X_{k}+\Omega_{k}=\Psi_{k}+Y_{k} .
$$

Note that the budget consists of the beginning money and revenue. Then, it is disposed only as expenditure and the end money. Accordingly, Equation (1) can also be interpreted as a budget constraint. This seems to be the most simple budget constraint.

Suppose that budgets of any agent are all expended under this constraint. In this case, $\Omega_{k}=0$ holds for $\forall k \in N$. That is, all portions of the end money of any agent are zeroes. If this supposition is realized, money does not disappear and is not transferred to the outside. Therefore, money ought to exist in the relevant spacetime. Based on common sense, money seems to exist somewhere at the term end in this case, but it is also denied because a zero value for end money is supposed.

Then, where does money exist? Money in the relevant space-time is all missing. This situation is reasonable if all budgets are zeroes, but it also occurs even if some budgets are positive. Such a situation seems to be unreasonable. However, as far as agents expend under the simple budget constraint, this unreasonable situation can occur. We call this the first missing problem of the monetary budget constraint. This problem shows that the simple budget constraint is incorrect as an objective budget constraint of a monetary economy.

There is a possibility that all agents subjectively plan to expend their total budgets. However, based on the unreasonableness of this, as shown in the discussion of the first missing problem, we cannot believe that all of their plans are objectively realized. At least one agent is sure to make a wrong plan and thus be forced to modify it. This is an example of why we should not ignore the objective constraint even if we consider subjective decision making by agents.

Then, where did we make a mistake? Two possibilities can be assumed. The first possibility is that the simple budget constraint does not hold. However, the equality between the sum of gross source and that of gross result is a basic fact of a monetary economy, thus we cannot believe it does not hold. Therefore, we should examine the second possibility. Even if the simple budget constraint holds, it may not be a unique constraint for expenditure. There remains a possibility that a hidden constraint exists and it may solve the problem of missing money.

"Hidden" in the sense used here means that the constraint is not reflected in the formalized budget constraint. It does not mean that it cannot be observed empirically. If we turn our eyes to an empirical monetary economy, we can find that expenditure behavior of economic agents is constrained by various conditions.

We have to sleep periodically in order to live. It is a basic bodily condition of human beings. While we sleep, we cannot expend. Therefore, the bodily condition that we have to sleep is a constraint for expenditure. Furthermore, we have to move some parts of our body to trade. Someone who is incapacitated may have difficulty in trading. This is also a bodily condition which constitutes a constraint for expenditure.

Also, if we want to buy a commodity in a certain store, we must visit the store. It is difficult to buy a commodity sold only in a distant store. Not only simple distance, but also the maintenance condition of roads and bridges affects the possibility of movement to the store. That is, a geographical condition is also a constraint for expenditure. We can also regard climate as one of the geographical conditions.

Even if a bodily handicap exists or a trading partner is in a distant location, trading is possible when supported by the means of communication. But viewed from the opposite side, this highlights a difficulty of trading without these means. Hence, a communication condition is also a constraint for expenditure.

Further, if commonly intelligible language between agents does not exist, it is difficult to communicate for trading and trading is difficult to realize. Thus, a linguistic condition is also a constraint for expenditure.

Moreover, trading is often blocked by political powers. A political condition is also a constraint for expenditure.

These constraints proceed from human environments ${ }^{5}$, thus we can call them collectively environmental constraints. Human beings can live and expend money only under these constraints. Nevertheless, they are not reflected in the simple budget constraint. The solution to the missing problem may depend on the existence of these constraints.

It is sure that the environmental constraints exist qualitatively, but to quantify them is not an easy work. However, the focus of this study is not what kind of constraint exists but what kind of constraint can solve the first missing problem. What we have to quantify is a constraint which answers the purpose. Note that most of the environmental constraints are variable personally or regionally or historically. On the other hand, the impossibility of the missing of money seems to be a universal truth of a monetary economy. Therefore, the constraint to pro-

$\overline{5}$ If we regard mind as an essence of a human being, body is their first environment. 
tect it must be universal. Environmental constraints which are valid only in specific situations are not suitable for a solution of the problem, thus we do not have to consider them for our purpose. We dedicate ourselves to search for a universal constraint for expenditure.

\section{Reflux Budget Constraint and the Second Missing Problem of the Monetary Budget Constraint}

Is there a universal monetary truth which is not yet reflected in the simple budget constraint?

Here, we will consider the essence of a monetary economy. In a monetary economy, revenue included in the budget is generated by the expenditure of others. Moreover, if we trace the sources of others' expenditure, a portion of them comes from their revenue. A portion of the revenue proceeds from the expenditure of the original agent. Eventually, part of the money expended by an agent should return as their own revenue. In Miura [2], we called this phenomenon the expenditure reflux.

Note that the simple budget constraint represents a kind of relationship between revenue and expenditure. It refers to a flow from revenue to expenditure 6 , but money cannot circulate only by this flow. In order to circulate, a flow from expenditure to revenue is also necessary. The latter flow is nothing but the expenditure reflux. Only after incorporating the reflux, the budget constraint can express money circulation perfectly. Nevertheless, the simple budget constraint never incorporates it. Therefore, the simple constraint does not reflect money circulation, which is essential to a monetary economy. This is a deficiency of the simple constraint.

We aim to incorporate the expenditure reflux into the budget constraint to express money circulation with the help of the quantification of the reflux shown in Miura [2].

Let $Z_{k j}$ be the separate transfer quantity from Agent $k$ to Agent $j$. From the perspective of Agent $k$, it refers to the separate expenditure quantity. The following relationship holds between gross expenditure and separate expenditure.

$$
X_{k}=Z_{k 1}+Z_{k 2}+\cdots+Z_{k n} .
$$

Furthermore, let $\varepsilon_{k h k}$ be the expenditure reflux coefficient of Agent $k$ through Agent $h$. This represents the quantity which refluxes as revenue per unit increase of the separate expenditure of Agent $k$ to Agent $h$. It is defined by the expenditure rate and the expenditure distribution rate of all agents other than Agent $k^{7}$. The expenditure reflux formula, which is a quantification of the expenditure reflux, is as follows ${ }^{8}$ :

$$
Y_{k}=Z_{k k}+\sum_{h \neq k} \varepsilon_{k h k}\left(Z_{k h}+\Psi_{h}\right) .
$$

Substituting Equations (2) and (3) in Equation (1), we can derive

$$
\sum_{j \in N} Z_{k j}+\Omega_{k}=\Psi_{k}+Z_{k k}+\sum_{h \neq k} \varepsilon_{k h k}\left(Z_{k h}+\Psi_{h}\right) .
$$

Rearranging this equation, we can derive

$$
\sum_{h \neq k}\left(1-\varepsilon_{k h k}\right) Z_{k h}+\Omega_{k}=\Psi_{k}+\sum_{h \neq k} \varepsilon_{k h k} \Psi_{h} .
$$

Equation (4) is a budget constraint which incorporates the expenditure reflux into the simple constraint. We call this the reflux budget constraint. This constraint is superior to the simple constraint at the point that it reflects both flows of which money circulation consists. Moreover, it includes the end money of the others $\left(\Psi_{h}\right)$ and the reflux coefficient $\left(\varepsilon_{k h k}\right)$. The latter is defined by the expenditure rate and the distribution rate of all others. Hence, the constraint reflects the situations of the others. This point is also a superiority of the reflux constraint.

The reflux budget constraint seems to be able to solve the first missing problem. We reconfirm that the expenditure reflux coefficient is limited to the following range ${ }^{9}$.

$$
0 \leq \varepsilon_{\text {khk }} \leq 1 \text {. }
$$

\footnotetext{
${ }^{6}$ The preceding paper has stated that the flow from revenue to expenditure is expressed by the circular disposal formula (Cf. Miura [2] p.27). This formula is derived from the law of gross disposal and the definition of the expenditure rate. The former is the same as the simple budget constraint in this paper, thus the simple constraint and the circular disposal formula are only the things which capture the same phenomenon from a slightly different angle.

${ }^{7}$ Cf. Equation (21) in Miura [2] p. 30.

${ }^{8}$ Cf. Equation (23) in Miura [2] p. 30.

${ }^{9} \mathrm{Cf}$. Theorem 3.6 in Miura [2] p. 32.
} 
The case $\varepsilon_{k h k}=1$ refers to a situation that the separate expenditure of Agent $k$ to Agent $h$ is fully returned to Agent $k$. We have defined this situation as the entire reflux of Agent $k$ through Agent $h^{10}$.

Note that the first missing problem occurs when all agents expend their total budgets. Based on this, we suppose that all agents excluding Agent $k$ expend their total budgets. This supposition is the same as assuming that $\Omega_{h}=0$ holds for $\forall h \neq k$. Miura [2] confirms that this is equivalent to the entire reflux of the gross expenditure occurring on Agent $k^{11}$. If we substitute this condition in the reflux budget constraint (Equation (4)), we obtain:

$$
\Omega_{k}=\sum_{h \in N} \Psi_{h}^{12} .
$$

We can see that if any agents excluding Agent $k$ expend their total budgets, all of the beginning money of the whole society concentrates in $k$. Note that this consequence is valid regardless of the level of expenditure of $k$. When $k$ expends any amounts of money, the end money of $k$ is always equal to the beginning money of the whole society.

This consequence shows that, even if any agents expend their budgets as much as possible, the end money is never missing provided that the beginning money exists somewhere in the relevant society. Hereby, the reflux budget constraint seems to have succeeded in solving the first missing problem.

Nevertheless, the problem is not completely solved. Note that the first missing problem was that all portions of the end money can be zeroes even if some budgets are positive. Budgets consist of the beginning money and revenue. In the case that some beginning money is positive, the problem has certainly been solved. However, in the case that any beginning money is zero and some revenue is positive, all of the end money can be zero by the assumption of the entire reflux and Equation (6). Hence, the problem has not been solved yet.

In the latter case, both the beginning money and the end money are equal to zero. This gives the impression that money does not exist in the relevant space-time. Nonetheless, revenue is still realized. This situation seems strange, but we have not obtained evidence of its impossibility.

In addition, another missing problem exists. Note that Equation (6) is originally the budget constraint. Nevertheless, this equation does not include expenditure. Therefore, if an agent expend as much as possible under this constraint, their expenditure become infinite.

If we suppose that the beginning money is an infinite quantity, it is a natural conclusion that expenditure is also infinite. Or if we put a supposition that the relevant term has an infinite length, infinite expenditure would also be no wonder. However, the conclusion is derived without these suppositions. It is not only unnatural but also an epistemological aporia because we cannot observe infinite expenditure empirically.

For the sake of normal recognition of a monetary economy, an upper limit of expenditure must exist, but the reflux budget constraint cannot guarantee the existence of this limit. In the constraint, an upper limit of expenditure is missing if the entire reflux occurs. We call this the second missing problem of the monetary budget constraint.

Looking back to the simple budget constraint based on this recognition, we notice that the finiteness of revenue is not guaranteed even if the beginning money is finite in the constraint. Hence, the finiteness of expenditure decided under the constraint is not guaranteed either. We can see that the simple budget constraint is also infected with the second missing problem.

Then, how can we solve this problem?

How about assuming that the entire reflux does not occur? If the reflux coefficient is not equal to unity, its range is $0 \leq \varepsilon_{k h k}<1$ by Equation (5) and the coefficient of expenditure in the reflux budget constraint (Equation (4)) is positive. In this case, expenditure has its upper limit when the beginning money is finite. Hence, the second missing problem does not appear.

However, can this assumption be justified in a monetary economy? In reality, most economic agents tend to retain some money in hand. Therefore, the assumption of the impossibility of entire reflux may be valid in a practical economic analysis. But the assumption that all portions of others' end money are equal to zero seems not to be prohibited by any universal law, even though this is a rare case. Given that there is a little possibility of this assumption, we should not ignore it.

Moreover, to deny an occurrence of entire reflux cannot be a sufficient solution to the second missing prob-

\footnotetext{
${ }^{10}$ Cf. Miura [2] p. 32.

${ }^{11}$ Cf. Theorem 3.9 in Miura [2] p. 33.

${ }^{12}$ This conclusion can also be derived from the framework of Miura [1] by using the law of gross disposal and the law of money conservation. Cf. Miura [1] p. 190.
} 
lem. In Miura [2], we define the transfer from an agent to oneself as the self-transfer ${ }^{13}$. In the preceding symbols, the self-transfer corresponds to $Z_{k k}$. The reflux budget constraint does not constrain self-transfer, regardless of the value of the reflux coefficient ${ }^{14}$. Self-transfer does not make sense if the constraint is that of an individual agent, but it makes sense if the constraint is that of a group of agents. We should not abandon a consideration of the latter case. Therefore, as far as the existence of constraints for self-transfer is not shown, it cannot be said that the authentic solution of the second missing problem has been obtained.

Eventually, we should judge that the reflux budget constraint is even incorrect as an authentic monetary budget constraint. In what way is the constraint incorrect?

Here, we should recall the solvability proof of the money circulation equation shown in Miura $[1]^{15}$. The existence of a unique solution to the equation means that expenditure can be calculated as a finite value. Then, that paper proves the solvability under the disposal irreversibility principle, which stipulates that money is only disposed from revenue of the past to expenditure of the future. Taking a contraposition of this, if expenditure is infinite, money is disposed time reversibly. If we apply this finding to the second missing problem, we must conclude that the reflux budget constraint permits a time reversible disposal.

Examining the simple budget constraint (Equation (1)), we tend to interpret that past revenue is disposed to future expenditure. Conversely, when we gaze at the expenditure reflux formula (Equation (3)), we tend to interpret that past expenditure refluxes to future revenue.

However, such interpretations do not have any mathematical basis because any temporal relationships are not directly described in the equations. Mathematically, the former constraint still permits a disposal from future revenue to past expenditure, and the latter formula still permits a reflux from future expenditure to past revenue. Since the reflux budget constraint is derived from the two equations, it also permits a time reversible circulation.

Here, the essence of the second missing problem becomes apparent. This problem and the solvability problem of the money circulation equation seems to be the same problems grasped from different viewpoints. The cause of the second missing problem is sure to proceed from the lack of the consideration of time irreversible disposal. As is the case for the solvability problem of the money circulation equation, the second missing problem is presumed to be solved if we incorporate the disposal irreversible principle into the budget constraint.

Previously, we pointed out a strange situation which can occur in the case of entire reflux. It was a situation in which revenue is positive even though the beginning money and the end money are both zero. We guessed that this situation shows that revenue can be realized even if money does not exist, but this guess is wrong in the case that time reversible disposal is permitted. In this case, money can circulate infinitely in a temporally closed place. Therefore, money can exist even if the beginning money and the end money are both zero. We tend to think that this situation is strange, but such a tendency is formed because we usually make the subconscious assumption that money is disposed time irreversibly ${ }^{16}$.

Furthermore, in Miura [1], we have proved that time irreversible disposal causes the space-time openness condition for the result direction ${ }^{17}$. The condition includes that the end money of at least one agent is positive. Hence, the first missing problem is also presumed to be solved considering time irreversibility.

A time reversible disposal of money is substantially the same as supposing that money can travel into the past. There are some physicists researching the possibility of time travel, but they only discuss its logical possibility at present. Any reliable evidence that this logical possibility can be realistic has never been shown until today ${ }^{18}$.

At the end of the preceding section, we have declared that we have to inquire into a universal monetary budget constraint for the solution of the first missing problem. The disposal irreversibility principle can be regarded as one of environmental constraints, but it can possibly be a universal constraint differently from other environmental constraints mentioned in the preceding section. A constraint by time irreversible disposal seems to be suitable also from this point.

We have confirmed that the irreversibility budget constraint is a strong candidate for solving the missing problems. Then, we aim to construct the solution.

\footnotetext{
${ }^{13}$ Cf. Miura [2] p. 31.

${ }^{14}$ Note that, as mentioned in Miura [2] p. 31, we can interpret that the expenditure reflux coefficient of the self-transfer is always equal to unity.

${ }^{15}$ Cf. Miura [1] pp. 192-197.

${ }^{16}$ The discussion regarding the jinn particle in a physics world may promote an understanding of this issue. Cf. Lossev \& Novikov [4], Gott [5] pp. 20-24.

${ }^{17}$ Cf. Theorem 3.5 in Miura [1] pp. 195-197.

${ }^{18}$ Cf. Gott [5], Davies [6], Nemiroff \& Wilson [7].
} 


\section{Irreversibility Budget Constraint and a Provisional Solution of the Missing Problems}

First, we divide the relevant term into $t$ subterms. However, we set the index of subterms such that a smaller index corresponds to an earlier subterm and a larger index corresponds to a later subterm. Moreover, we express the beginning of the relevant term as Subterm 0 and the end of the relevant term as Subterm \#. We denote the relevant term including the term beginning and the term end as $T=\{0,1,2, \cdots, t$,\# .

Expenditure in Subterm $u$ is defined as transferring money within Subterm $u$. Moreover, revenue into Subterm $u$ is defined as money being transferred into Subterm $u$. Let $X_{k(u)}$ be the expenditure quantity of Agent $k$ in Subterm $u$, and $Y_{k(u)}$ be the revenue quantity of Agent $k$ in Subterm $u$. Money transfer is the quantities defined only in the relevant term, thus $X_{k(u)}$ and $Y_{k(u)}$ are defined only in the range $1 \leq u \leq t$.

The beginning money consists of the term beginning possession, production and being transferred from the outside. We decide that $\Psi_{k(0)}$ denotes the quantity of the term beginning possession, and $\Psi_{k(u)}$ for $1 \leq u \leq t$ denotes the quantity of production and being transferred from the outside in Subterm $u$. Further, the end money consists of the term end possession, disappearance and transferring to the outside. We decide that $\Omega_{k(u)}$ for $1 \leq u \leq t$ denotes the quantity of disappearance and transferring to the outside in Subterm $u$, and $\Omega_{k(\#)}$ denotes the quantity of the term end possession.

We define the disposal as a process from source to result. Let $\delta_{k(p q)}$ be the disposal quantity that Agent $k$ disposed from sources in Subterm $p$ to results in Subterm $q$. From their economic meaning, they are all non-negative. That is, $\delta_{k(p q)} \geq 0$ holds for $\forall k \in N, \forall p, q \in T$.

The relationships between the disposal quantities and others are summarized in the following disposal table.

\begin{tabular}{|c|c|c|c|c|c|c|}
\hline $\begin{array}{ll} & \text { Result } \\
\text { Source } & \end{array}$ & Subterm 1 & Subterm 2 & $\cdots$ & Subterm $t$ & Term End & Sum \\
\hline Term Beginning & $\delta_{k(01)}$ & $\delta_{k(02)}$ & $\ldots$ & $\delta_{k(0 t)}$ & $\delta_{k(0 *)}$ & $\Psi_{k(0)}$ \\
\hline Subterm 1 & $\delta_{k(11)}$ & $\delta_{k(12)}$ & $\cdots$ & $\delta_{k(1 t)}$ & $\delta_{k(1 \#)}$ & $\Psi_{k(1)}+Y_{k(1)}$ \\
\hline Subterm 2 & $\delta_{k(21)}$ & $\delta_{k(22)}$ & $\cdots$ & $\delta_{k(2 t)}$ & $\delta_{k(2 \#)}$ & $\Psi_{k(2)}+Y_{k(2)}$ \\
\hline$\cdots$ & $\cdots$ & $\cdots$ & $\cdots$ & $\cdots$ & $\cdots$ & $\cdots$ \\
\hline Subterm $t$ & $\delta_{k(t 1)}$ & $\delta_{k(t 2)}$ & $\cdots$ & $\delta_{k(t)}$ & $\delta_{k(t \#)}$ & $\Psi_{k(t)}+Y_{k(t)}$ \\
\hline Sum & $X_{k(1)}+\Omega_{k(1)}$ & $X_{k(2)}+\Omega_{k(2)}$ & $\cdots$ & $X_{k(t)}+\Omega_{k(t)}$ & $\Omega_{k_{(*)}}$ & \\
\hline
\end{tabular}

Here, we define the disposal irreversibility principle precisely. It represents that money can be only disposed from the sources of the past to the results of the future. By this principle, a disposal quantity from the future to the past is naturally zero. Therefore, $\delta_{k(p q)}=0$ holds for $\forall k \in N, \forall p>q$.

We cannot judge whether a simultaneous disposal quantity is positive or zero generally. However, if it is positive even in the case we divide the relevant term into infinitesimally short subterms, it contradicts the disposal irreversibility principle because this principle implies that money cannot be disposed at exactly the same time. Therefore, if we divide it into exceedingly short subterms, any simultaneous disposal quantity must be zero. The length of this short subterm may be different for different agents. We define a basic-term as this short subterm in which simultaneous disposal quantities of all agents are zero. Since the relevant term is finite length, the number of basic-terms included in the relevant term is also finite ${ }^{19}$.

Let Subterm $u$ be a basic-term. Moreover, let Subterm - be a subterm which is before Basic-term $u$, and Subterm + be a subterm which is after Basic-term $u$. By the disposal irreversibility principle, the disposal table of Agent $k$ is as follows.

\footnotetext{
${ }^{19}$ In Footnote 11 in Miura [1] p. 195, we have derived the finiteness of the number of basic-terms from the finiteness of the number of disposals. But in this paper, since we have shown the possibility of the infinity of expenditure as the second missing problem, introducing the finiteness of the number of disposal beforehand seems to be inappropriate. Therefore, this paper assumes that a basic-term is finite length. Due to this assumption, the finiteness of the number of basic-terms is justified because the relevant term, which is the sum of basic-terms, is of finite length.
} 


\begin{tabular}{|c|c|c|c|c|c|}
\hline Source Result & Subterm - & Basic-term $u$ & Subterm + & Term End & Sum \\
\hline Term Beginning & $\delta_{k(0-)}$ & $\delta_{k(0 u)}$ & $\delta_{k(0+)}$ & $\delta_{k(0 \#)}$ & $\Psi_{k(0)}$ \\
\hline Subterm - & $\delta_{k(-)}$ & $\delta_{k(-u)}$ & $\delta_{k(-+)}$ & $\delta_{k(-\#)}$ & $\Psi_{k(-)}+Y_{k(-)}$ \\
\hline Basic-term $u$ & 0 & 0 & $\delta_{k(u+)}$ & $\delta_{k(u \#)}$ & $\Psi_{k(u)}+Y_{k(u)}$ \\
\hline Subterm + & 0 & 0 & $\delta_{k(++)}$ & $\delta_{k(+*)}$ & $\Psi_{k(+)}+Y_{k(+)}$ \\
\hline Sum & $X_{k(-)}+\Omega_{k(-)}$ & $X_{k(u)}+\Omega_{k(u)}$ & $X_{k(+)}+\Omega_{k(+)}$ & $\Omega_{k(*)}$ & \\
\hline
\end{tabular}

Then, we will define hoarding of money based on this framework. Note that usual usage of hoarding includes two meanings. One is money which is disposable but is not disposed, and the other is money which is retained in the possession of an agent. These two meanings are not usually distinguished because they are regarded to represent the same thing. However, considering a time irreversible disposal, they have different meanings. We define hoarding based on the former meaning.

Based on the disposal irreversibility principle, disposable money till Basic-term $u$ is as follows:

$$
\Psi_{k(0)}+\Psi_{k(-)}+Y_{k(-)}=\delta_{k(0-)}+\delta_{k(0 u)}+\delta_{k(0+)}+\delta_{k(0 \#)}+\delta_{k(--)}+\delta_{k(-u)}+\delta_{k(-+)}+\delta_{k(-\#)} .
$$

Among these disposal quantities, $\delta_{k(0-)}, \delta_{k(0 u)}, \delta_{k(--)}$ and $\delta_{k(-u)}$ are disposed until Basic-term $u$. Therefore, disposal quantities which are disposable but are not disposed till $u$ are $\delta_{k(0+)}, \delta_{k(0 \#)}, \delta_{k(-+)}$ and $\delta_{k(-\#)}$. Based on this recognition, we define a hoarding quantity of Agent $k$ in Basic-term $u$ as follows:

$$
H_{k(u)}=\delta_{k(0+)}+\delta_{k(0 \#)}+\delta_{k(-+)}+\delta_{k(-\#)} \text {. }
$$

$H_{k(u)}$ corresponds to an upper right corner of $\delta_{k(u u)}$ in the disposal table. It is non-negative because of the non-negativity of disposal quantities.

Further, let $\Pi_{k(u ; u+1)}$ be the quantity of money which is possessed at the end of Basic-term $u$ and at the be ginning of Basic-term $u+1$. It consists of disposal quantities which are obtained till Basic-term $u$ and disposed after Basic-term $u+1$. Considering this with the preceding table, the following relation holds.

$$
\Pi_{k(u ; u+1)}=\delta_{k(0+)}+\delta_{k(0 \#)}+\delta_{k(-+)}+\delta_{k(-\#)}+\delta_{k(u+)}+\delta_{k(u \#)}=H_{k(u)}+\Psi_{k(u)}+Y_{k(u)} .
$$

Due to Equation (8), we can see that hoarding in this paper is different from the retained money by as much as $\Psi_{k(u)}+Y_{k(u)}$.

From the preceding table and the definition of hoarding (Equation (7)), we can derive:

$$
X_{k(-)}+X_{k(u)}+\Omega_{k(-)}+\Omega_{k(u)}+H_{k(u)}=\Psi_{k(0)}+\Psi_{k(-)}+Y_{k(-)} .
$$

Based on this equation, we derive the budget constraint of each separate basic-term.

In the case $u=1$, Subterm - cannot be defined. Therefore, Equation (9) of the case $u=1$ is as follows:

$$
X_{k(1)}+\Omega_{k(1)}+H_{k(1)}=\Psi_{k(0)} .
$$

Based on the disposal irreversibility principle, $\Psi_{k(0)}$ is all of disposable budget of the first basic-term. Further, $X_{k(1)}, \Omega_{k(1)}$ and $H_{k(1)}$ refer to the contents of allocation of the budget in the first basic-term. Hence, Equation (10) is the budget constraint of Agent $k$ in the first basic-term.

Next, we think about the case $u \geq 2$. We line up Equation (9) of $u-1$ and $u$ with embodying the content of Subterm - included in them.

$$
\begin{gathered}
\sum_{v=1}^{u-1} X_{k(v)}+\sum_{v=1}^{u-1} \Omega_{k(v)}+H_{k(u-1)}=\sum_{v=0}^{u-2} \Psi_{k(v)}+\sum_{v=1}^{u-2} Y_{k(v)} . \\
\sum_{v=1}^{u} X_{k(v)}+\sum_{v=1}^{u} \Omega_{k(v)}+H_{k(u)}=\sum_{v=0}^{u-1} \Psi_{k(v)}+\sum_{v=1}^{u-1} Y_{k(v)} .
\end{gathered}
$$


Subtracting both sides of them and rearranging them, we obtain:

$$
X_{k(u)}+\Omega_{k(u)}+H_{k(u)}=H_{k(u-1)}+\Psi_{k(u-1)}+Y_{k(u-1)} .
$$

Note that the following equation is satisfied due to Equation (8).

$$
H_{k(u-1)}+\Psi_{k(u-1)}+Y_{k(u-1)}=\Pi_{k(u-1 ; u)} .
$$

We can see that the quantity in the right side of Equation (11) refers to the possessed money at the beginning of Basic-term $u$. Based on the disposal irreversibility principle, $\Pi_{k(u-1 ; u)}$ is all of disposable budget of Basic-term $u$. Further, $X_{k(u)}, \Omega_{k(u)}$ and $H_{k(u)}$ refer to the contents of allocation of the budget in Basic-term $u$. Therefore, Equation (11) is the budget constraint of Agent $k$ in the following basic-term.

Note that the subterm of revenue and that of expenditure in Equation (11) are different. This is a key point of this constraint. If those are simultaneous, the possibility of time reversible disposal cannot be eliminated. By the difference of those subterms, the inevitability of a time irreversible disposal is guaranteed.

We have finished clarifying the budget constraint in every basic-term, and we shall now clarify how the term end possession is decided.

Let Subterm-be the set of basic-terms before Basic-term $t$. The disposal table considering the disposal irre-

\begin{tabular}{|c|c|c|c|c|}
\hline $\begin{array}{ll}\text { Source } & \text { Result } \\
\end{array}$ & Subterm - & Basic-term $t$ & Term End & Sum \\
\hline Term Beginning & $\delta_{k(0-)}$ & $\delta_{k(0 t)}$ & $\delta_{k(0 *)}$ & $\Psi_{k(0)}$ \\
\hline Subterm - & $\delta_{k(-)}$ & $\delta_{k(-t)}$ & $\delta_{k\left(-H^{*}\right)}$ & $\Psi_{k(-)}+Y_{k(-)}$ \\
\hline Basic-term $t$ & 0 & 0 & $\delta_{k(t+t)}$ & $\Psi_{k(t)}+Y_{k(t)}$ \\
\hline Sum & $X_{k(-)}+\Omega_{k(-)}$ & $X_{k(t)}+\Omega_{k(t)}$ & $\Omega_{k(f)}$ & \\
\hline
\end{tabular}
versibility principle is as follows.

From this table, we can see that:

$$
\begin{aligned}
\Omega_{k(\#)}= & \delta_{k(0 \#)}+\delta_{k(-\#)}+\delta_{k(t \#)} . \\
& \Psi_{k(t)}+Y_{k(t)}=\delta_{k(t \#)} .
\end{aligned}
$$

Moreover, since Subterm + cannot be defined in this case, we obtain the following equation from the definition of hoarding (Equation (7)).

$$
H_{k(t)}=\delta_{k(0 \#)}+\delta_{k(-\#)} .
$$

From Equations (12), (13) and (14), we can derive:

$$
\Omega_{k(\#)}=H_{k(t)}+\Psi_{k(t)}+Y_{k(t)} .
$$

We call Equation (15) the term end settlement formula.

The irreversibility budget constraint is a set of the budget constraints of all basic-terms and the settlement formula. If we add up the budget constraints of all basic-terms and the settlement formula, we can derive the following equation:

$$
\sum_{u \in T} X_{k(u)}+\sum_{u \in T} \Omega_{k(u)}+\sum_{u \in T} H_{k(u)}=\sum_{u \in T} H_{k(u)}+\sum_{u \in T} \Psi_{k(u)}+\sum_{u \in T} Y_{k(u)} .
$$

Removing $\sum_{u \in T} H_{k(u)}$ from both sides of this equation, we obtain:

$$
\sum_{u \in T} X_{k(u)}+\sum_{u \in T} \Omega_{k(u)}=\sum_{u \in T} \Psi_{k(u)}+\sum_{u \in T} Y_{k(u)} .
$$

This is nothing but the simple budget constraint. We can see that the simple constraint is included within the irreversibility budget constraint.

The irreversibility budget constraint itself is insufficient to solve the missing problems. Suppose that 
$X_{k(1)}=\Psi_{k(0)}>0$ for $\forall k \in N$ and $\Psi_{k(u)}=Y_{k(u)}=0$ for $\forall k \in N, \forall u \in T \backslash\{0\}$. In this case, we can derive $\Omega_{k(u)}=H_{k(u)}=0$ for $\forall k \in N, \forall u \in T^{20}$, thus the first missing problem is not resolved. Further, the possibility of infinite revenue is not still eliminated, thus expenditure can be infinite in the case that the relevant term includes plural basic-terms. That is, the second missing problem is not resolved either.

What is lacking? We should recall the solvability proof of the money circulation equation in Miura [1]. For the proof, we required not only time irreversible disposal but also a simultaneous relationship between expenditure and revenue. The reason why the latter relationship is required seems to be that it is necessary to represent the effect of money circulation. Also for the solution of the missing problems, we seem to need to consider this relationship, too.

Let $Z_{i j(u)}$ be the separate transfer quantity from Agent $i$ to Agent $j$ in Subterm $u$. Based on the definitions of expenditure and revenue, the following relations holds for all agents and all basic-terms.

$$
\begin{aligned}
& Z_{i 1(u)}+Z_{i 2(u)}+\cdots+Z_{i n(u)}=X_{i(u)} . \\
& Z_{1 j(u)}+Z_{2 j(u)}+\cdots+Z_{n j(u)}=Y_{j(u)} .
\end{aligned}
$$

From these equations, we obtain:

$$
X_{1(u)}+X_{2(u)}+\cdots+X_{n(u)}=Y_{1(u)}+Y_{2(u)}+\cdots+Y_{n(u)} .
$$

We call Equation (16) the law of transfer equality in each subterm ${ }^{21}$. This law reflects the fact that expenditure and revenue are the same entity grasped from different viewpoints. By considering both this law and the irreversibility constraint, we will show a provisional solution of the missing problems.

First, we show a provisional solution of the second missing problem. The same as the preceding section, we put a premise that the beginning money is finite.

Lemma 4.1. Expenditure and hoarding in each basic-term is finite.

Proof. We prove this by mathematical induction.

By the budget constraint of the first basic-term (Equation (10)) and the non-negativity of $\Omega_{k(1)}$ and $H_{k(1)}$, we can see that $X_{k(1)} \leq \Psi_{k(0)}$ is satisfied. Since the finiteness of the beginning money is premised, $X_{k(1)}$ is also finite. The finiteness of $H_{k(1)}$ can be proved by a similar way.

Next, we think about the case $u \geq 2$. Suppose that $X_{k(u-1)}$ and $H_{k(u-1)}$ are both finite. Based on Equation (11), we add up the budget constraints of any agents in Basic-term $u$. The following equation can be obtained:

$$
\sum_{k \in N} X_{k(u)}+\sum_{k \in N} \Omega_{k(u)}+\sum_{k \in N} H_{k(u)}=\sum_{k \in N} H_{k(u-1)}+\sum_{k \in N} \Psi_{k(u-1)}+\sum_{k \in N} Y_{k(u-1)} \text {. }
$$

Considering this with the non-negativity of $\sum_{k \in N} \Omega_{k(u)}$ and $\sum_{k \in N} H_{k(u)}$, we can see that

$$
\sum_{k \in N} X_{k(u)} \leq \sum_{k \in N} H_{k(u-1)}+\sum_{k \in N} \Psi_{k(u-1)}+\sum_{k \in N} Y_{k(u-1)}
$$

is satisfied. The finiteness of $\sum_{k \in N} \Psi_{k(u-1)}$ is guaranteed from the premise of the finiteness of the beginning money. The finiteness of $\sum_{k \in N} H_{k(u-1)}$ is a supposition of mathematical induction. The finiteness of $\sum_{k \in N} Y_{k(u-1)}$ is derived from the supposition that $\sum_{k \in N} X_{k(u-1)}$ is finite and the law of transfer equality in Basic-term $u-1$ (Equation (16)). Therefore, the right side of Equation (17) is finite. From this, the finiteness of $\sum_{k \in N} X_{k(u)}$, which is included in the left side of Equation (17), is derived. Due to the non-negativity of expenditure, each $X_{k(u)}$ is also finite. The finiteness of $H_{k(u)}$ can be proved by a similar way [Q.E.D.].

Theorem 4.2. Expenditure in the relevant term is finite.

Proof. Expenditure in each basic-term is a finite quantity by Lemma 4.1 and the relevant term consists of a finite basic-term. The finite sum of finite quantities is finite [Q.E.D.].

\footnotetext{
${ }^{20}$ This proposition can be derived by Equations (10), (11) and (15) with a mathematical induction.

${ }^{21}$ Cf. Miura [1] p. 194.
} 
By Theorem 4.2, we have succeeded in solving the second missing problem.

Next, we will show a provisional solution of the first missing problem. For the purpose, we prove the next theorem.

Theorem 4.3. If $\exists k \in N, \exists u \in T$ such that $\Psi_{k(u)}>0$ or $Y_{k(u)}>0$ or $H_{k(u)}>0, \exists k \in N, \exists v \in T$ such that $\Omega_{k(v)}>0$.

Proof. Based on the premise, let Agent $k$ be the agent either whose beginning money or revenue or hoarding is lastly positive in the relevant society. Moreover, let Basic-term $u$ be the last basic-term in which the beginning money or revenue or hoarding of Agent $k$ is positive ${ }^{22}$.

We first examine the case $u<t$. Due to the budget constraint in Basic-term $u+1$ and the definition of Basic-term $u$ that it is the last basic-term in which the beginning money or revenue or hoarding is positive in the relevant society, we can see that:

$$
X_{k(u+1)}+\Omega_{k(u+1)}+H_{k(u+1)}=H_{k(u)}+\Psi_{k(u)}+Y_{k(u)}>0
$$

Also from the definition of Basic-term $u, Y_{k(u+1)}=H_{k(u+1)}=0$ holds for $\forall k \in N$. Due to the law of transfer equality (Equation (16)), $Y_{k(u+1)}=0$ for $\forall k \in N$ derives $X_{k(u+1)}=0$ for $\forall k \in N$. Eventually, $X_{k(u+1)}=H_{k(u+1)}=0$ holds for $\forall k \in N$. Applying this condition to Equation (18), $\Omega_{k(u+1)}>0$ is derived.

We next examine the case $u=t$. Due to the term end settlement formula (Equation (15)),

$\Omega_{k(\#)}=H_{k(t)}+\Psi_{k(t)}+Y_{k(t)}$ holds. By the definition of Agent $k$ and Basic-term $u=t$, the right side of this equation is positive. Hence, we can derive $\Omega_{k(\#)}>0$.

We have proved that $\exists k \in N, \exists v \in T$ such that $\Omega_{k(v)}>0$ in any case [Q.E.D.].

The budget in every basic-term consists of the beginning money, revenue and hoarding as shown in Equations (10) and (11). Hence, the premise of Theorem 4.3 implicates a positivity of the budget. Then, this theorem concludes that the end money of at least one agent is positive. Hereby, the first missing problem has been solved.

Note that the budget constraint considering time irreversible disposal is already known under the name of finance constraint. It was suggested by Sho Chieh Tsiang inspired by Dennis Holme Robertson ${ }^{23}$. The concept of a "day" used by Robertson is equivalent to the "basic-term" concept used in this paper. Our additional contributions are having given a rigorous foundation for the constraint using the disposal quantity and having shown the logical significance of the irreversibility constraint through solving the two missing problems.

The cash-in-advance model is a more systematic theory which is relevant to the finance constrain ${ }^{24}$. It is often said to have been first suggested by Robert Wayne Clower $^{25}$, but Mauro Boianovsky reported that it was already suggested by Mario Henrique Simonsen in Brazil before Clower ${ }^{26}$.

We confirm the basic cash-in-advance model according to Robert Emerson Lucas. Using the symbols used in this paper, it consists of the following two constraints:

$$
\begin{gathered}
\Omega_{k(\#)}=\Psi_{k(0)}-X_{k}+Y_{k}, \\
\Psi_{k(0)} \geq X_{k} .
\end{gathered}
$$

Lucas called the former the standard budget constraint, and called the latter the cash-in-advance constraint ${ }^{27}$.

Suppose that the relevant term consists of one basic-term in our framework. Also suppose that production and disappearance of money does not occur in the relevant term, and money is not transferred between the relevant society and its outside, either. In this case, we can derive the latter cash-in-advance constraint from our budget constraint of the first basic-term (Equation (10)) and the non-negativity of hoarding. Moreover, the former standard budget constraint can be interpreted as our simple budget constraint, which can be derived from adding up our budget constraint in each basic-term and our term end settlement formula. Therefore, Lucas' cash-in-advance model can be interpreted as a special case of our irreversibility budget constraint where the relevant term consists of one basic-term.

\footnotetext{
${ }^{22}$ The existence of the last basic-term is guaranteed because the relevant term consists of finite basic-terms.

${ }^{23}$ Cf. Robertson [8], Keynes \& Hawtrey \& Robertson [9], Metzler [10], Tsiang [11], Kohn [12], Kohn [13], Laidler [14], Van Eeghen [15]. Note that Robertson reported a Spanish pioneer of his idea named Germán Bernácer. Cf. Robertson [16], Boianovski \& Dar \& Presley \& Garza [17]. The author owes a knowledge of the finance constraint largely to Ohara [18] and Ohara [19] written in Japanese.

${ }^{24} \mathrm{Cf}$. Kohn [13] pp. 5-8 regarding relevance between the finance constraint and the cash-in-advance model.

${ }^{25}$ Cf. Clower [20], Lucas [21], Howitt [22], Howitt [23], Walsh [24] pp. 95-118.

${ }^{26}$ Cf. Boianovsky [25].

${ }^{27}$ Cf. Lucas [21] pp. 205-206.
} 
As pointed out in the preceding section, we have needed the help of the law of transfer equality to solve the missing problems. However, Lucas' cash-in-advance model does not include this law. Hence, it cannot eliminate the possibility of causing the first missing problem ${ }^{28}$. This is a weakness of this model.

\section{Concluding Comments}

This paper has inquired into an authentic monetary budget constraint. First, we show that the simple budget constraint cannot avoid the first missing problem. We construct the reflux budget constraint to solve the problem, but it has a possibility to cause the second missing problem. Referring to the solvability proof of the money circulation equation, we clarify that the irreversibility budget constraint gives a provisional solution of the missing problems.

The irreversibility constraint can solve the second missing problem, whereas the reflux constraint cannot. Therefore, some may think that the former is more valid than the latter. However, we should notice that the solution of the missing problems by the irreversibility constraint depends on the law of transfer equality, which exists outside of the constraint. Ideally, the problems should be solved only inside of a constraint. This is a reason why we describe the solution as "provisional". In order to solve the missing problems perfectly, we must show the solution based on a budget constraint which incorporates an effect of money circulation in its inside.

Note that the reflux constraint reflects money circulation, whereas the irreversibility constraint does not. For the perfect solution of the missing problems, we ought to construct a constraint which reflects both time irreversible disposal and expenditure reflux. This irreversibility reflux budget constraint is a more authentic constraint of a monetary economy. In this constraint, revenue of each subterm has to be denoted as a function of expenditure in each subterm. For this purpose, we have to invent a time division type money circulation equation, in which any quantity is divided into every subterm. However, the author has not invented the equation, thus he cannot yet show a general irreversibility reflux budget constraint.

Nevertheless, note the expenditure reflux formula (Equation (3)) in the case that the relevant society includes only one agent: $Y_{1}=Z_{11}=X_{1}$. This is the same as the law of transfer equality. That is, the expenditure reflux formula of the whole society is equivalent to the law of transfer equality. This law directly reflects a fact that expenditure and revenue are the same entity, but it can also be interpreted as an expression of the flow from expenditure to revenue grasped from the viewpoint of the whole society.

Accordingly, the irreversibility reflux constraint of the whole society can be constructed without the time division type money circulation equation. It can be derived only substituting the law of transfer equality in the irreversibility constraint. The missing problems of this whole irreversibility reflux constraint have already been solved because the provisional solution of the missing problems by the irreversibility constraint is obtained by considering the law of transfer equality.

Based on the whole irreversibility reflux constraint, the author will discuss a money circulation optimization theory, which synthesizes a mathematical money circulation analysis and an optimization method of human behavior, in the near future.

\section{References}

[1] Miura, S. (2014) Money Circulation Equation Considering Time Irreversibility. Advances in Linear Algebra \& Matrix Theory, 4, 187-200. http://dx.doi.org/10.4236/alamt.2014.44016

[2] Miura, S. (2015) Quantification of Revenue Induction and Expenditure Reflux in a Monetary Economy. Advances in Linear Algebra \& Matrix Theory, 5, 25-35. http://dx.doi.org/10.4236/alamt.2015.51003

[3] Christ, C.F. (1968) A Simple Macroeconomic Model with a Government Budget Restraint. Journal of Political Economy, 76, 53-67. http://dx.doi.org/10.1086/259381

[4] Lossev, A. and Novikov, I.D. (1992) The Jinn of the Time Machine: Non-Trivial Self-Consistent Solutions. Classical and Quantum Gravity, 9, 2309-2321. http://dx.doi.org/10.1088/0264-9381/9/10/014

[5] Gott, J.R. (2002) Time Travel in Einstein's Universe: The Physical Possibilities of Travel through Time. Phoenix, London.

[6] Davies, P. (2002) How to Build a Time Machine. Penguin, London.

[7] Nemiroff, R.J. and Wilson, T. (2013) Searching the Internet for Evidence of Time Travelers. arXiv:1312.7128.

\footnotetext{
${ }^{28}$ Since the finiteness of the unique expenditure is guaranteed by the cash-in-advance constraint, the second missing problem does not occur.
} 
http://arxiv.org/abs/1312.7128

[8] Robertson, D.H. (1933) Saving and Hoarding. The Economic Journal, 43, 399-413. http://dx.doi.org/10.2307/2224283

[9] Keynes, J.M., Hawtrey, R.G. and Robertson, D.H. (1933) Mr. Robertson on "Saving and Hoarding”. The Economic Journal, 43, 699-712. http://dx.doi.org/10.2307/2224530

[10] Metzler, L.A. (1948) Three Lags in the Circular Flow of Income. Income, Employment, and Public Policy: Essays in Honor of Alvin H. Hansen, W.W. Norton Company Inc., New York, 11-32.

[11] Tsiang, S.C. (1966) Warlas’ Law, Say’s Law and Liquidity Preference in General Equilibrium Analysis. International Economic Review, 7, 329-345. http://dx.doi.org/10.2307/2525528

[12] Kohn, M. (1981) In Defense of the Finance Constraint. Economic Inquiry, 19, 175-195. http://dx.doi.org/10.1111/j.1465-7295.1981.tb01129.x

[13] Kohn, M. (1988) The Finance Constraint Theory of Money: A Progress Report. The Jerome Levy Economics Institute Working Paper, No. 5.

[14] Laidler, D. (1989) The Context of S.C. Tsiang’s Monetary Economics. In: Kohn, M., Ed., Finance Constraints and the Theory of Money: Selected Papers, Academic Press, San Diego, 359-375.

[15] Van Eeghen, P.H. (2014) Why DSGE Analysis Cannot Accurately Model Financial-Real Sector Interaction. RealWorld Economics Review, 67, 17-40. http://www.paecon.net/PAEReview/issue67/Eeghen67.pdf

[16] Robertson, D.H. (1940) A Spanish Contribution to the Theory of Fluctuations. Economica, 7, 50-65. http://dx.doi.org/10.2307/2549318

[17] Boianovsky, M., Dar, H., Presley, J.R. and Garza, P.B. (2006) Cambridge and the Spanish Connection: The Contribution of Germán Bernácer. History of Political Economy, 38, 407-436. http://dx.doi.org/10.1215/00182702-2006-001

[18] Ohara, H. (1998) Robertsonian Monetary Dynamic Theory as a Precursor to Finance Constraint Model: Monetary Theory with Different Direction from Keynes. Shakai Kagaku Kenkyu, 49, 91-245. (In Japanese)

[19] Ohara, H. (2000) Criticizing Meir Kohn’s Wicksell-Keynesian Monetary Model. Meidai Shogaku Ronsou, 82, $285-304$. (In Japanese) http://hdl.handle.net/10291/2078

[20] Clower, R.W. (1967) A Reconsideration of the Microfoundations of Monetary Theory. Economic Inquiry, 6, 1-8. http://dx.doi.org/10.1111/j.1465-7295.1967.tb01171.x

[21] Lucas Jr., R.E. (1980) Equilibrium in a Pure Currency Economy. Economic Inquiry, 18, 203-220. http://dx.doi.org/10.1111/j.1465-7295.1980.tb00570.x

[22] Howitt, P. (1992) Cash-in-Advance Economy. Vol. 1, In: Newman, P., Milgate, M. and Eatwell, J., Eds., The New Palgrave Dictionary of Money and Finance, Macmillan, London, 318-319.

[23] Howitt, P. (1996) Cash in Advance, Microfoundations in Retreat. In: Vaz, D. and Velupillai, K., Eds. Inflation, Institutions and Information: Essays in Honour of Axel Leijonhufvud, Macmillan, London, 62-88.

[24] Walsh, C.E. (2003) Monetary Theory and Policy. 2nd Edition, The MIT Press, Cambridge.

[25] Boianovsky, M. (2002) Simonsen and the Early History of the Cash-in-Advance Approach. The European Journal of the History of Economic Thought, 9, 57-71. http://dx.doi.org/10.1080/09672560110103388 